\title{
Endoplasmic reticulum stress links psoriasis vulgaris with keratinocyte inflammation
}

\author{
Min Zhao ${ }^{1,2}$, Jinqiang Luo ${ }^{1}$, Bin Xiao', Hongyan Tang', Fanjun Song ${ }^{1}$, Xiaolei Ding ${ }^{3}$, Guilan Yang ${ }^{1}$ \\ ${ }^{1}$ Department of Cosmo-dermatology, Shenzhen Hospital, Southern Medical University, Guangdong, China \\ 'Department of Rheumatism and Immunology, $5^{\text {th }}$ Hospital of Xi 'an, Xi'an, China \\ ${ }^{3}$ Department of Dermatology, University of Cologne, Cologne, Germany
}

Adv Dermatol Allergol 2020; XXXVII (1): 34-40 DOI: https://doi.org/10.5114/ada.2020.93382

\begin{abstract}
Introduction: Endoplasmic reticulum stress (ERS) has been implicated in the pathogenesis of various inflammatory diseases. However, the role of ERS in psoriasis is still unclear.

Aim: To examine ERS in psoriasis keratinocytes and to assess the association of ERS with skin inflammation response.

Material and methods: We investigated ERS in keratinocytes of normal skin, lesional and perilesional psoriasis vulgaris (PV) skin tissues using transmission electron microscope (TEM) examination, Western blot and immunostaining analysis.

Results: By TEM examination, we found that endoplasmic reticulum (ER) in psoriatic keratinocytes was ultrastructurally abnormal, with changes in ER morphology and the ER expansion. Using Western blot and immunostaining analysis, we showed that the expression of ERS-associated proteins, such as BiP, CHOP and XBP1, was enhanced in PV epidermis compared to the healthy skin. Moreover, abundant TNF- $\alpha$ protein was correlated to the increased $\mathrm{BiP}, \mathrm{CHOP}$ and XBP1 expression in PV epidermis.

Conclusions: Our findings demonstrate that PV keratinocytes have an increased ERS, which may contribute to the pathogenesis of PV.
\end{abstract}

Key words: endoplasmic reticulum, ER stress, psoriasis vulgaris, keratinocyte.

\section{Introduction}

Psoriasis is classified as a chronic, immune-mediated skin disease that affects approximately $2-3 \%$ of the population in the world [1]. There are five major subtypes of psoriasis: vulgaris (plaque), guttate, pustular, inverse, and erythrodermic, and psoriasis vulgaris (PV) represents the most common disease variant, constituting approximately $85-90 \%$ of psoriatic patients [2, 3]. The cause of psoriasis is very complex and it is primarily driven by aberrant immune response in combination with skin barrier disruption, genetic susceptibility and environmental stimuli [4]. The pathophysiology of PV is characterized by keratinocyte hyperproliferation and immune cell infiltration, mainly $T$ cells and dendritic cells (DCs) in the dermis $[5,6]$. The recent advances in the understanding of psoriasis pathogenesis have directly led to the development of highly effective therapeutic targets, such as against IL-17 and IL-23 [4]. Although it is clear that immune cells, such as T cells and DCs, play an important role in the pathogenesis of PV, the contribution of epidermal keratinocytes to the disease still remains poorly understood [7].

The endoplasmic reticulum (ER), a specialized cellular organelle is mainly responsible for protein homeostasis, including protein folding, maturation and protein quality control in the cells. Any condition that disturbs protein processing, particularly the accumulation of newly synthesized, unfolded proteins in ER lumen can lead to a state known as endoplasmic reticulum stress (ERS). In response to ERS, different signalling cascades are activated in the cells, evoking a stress response mechanism known as unfolded protein response (UPR) that aims to restore ER homeostasis [8]. Increasing evidence suggests that UPR intersects with many different inflammatory and stress signalling pathways, including the JNK-AP1 and NF-KB-IKK kinase pathways [9, 10]. The close link between ERS and inflammation is a likely contributor to

\footnotetext{
Address for correspondence: Xiaolei Ding PhD, Department of Dermatology, Cologne University, 50937 Cologne, Germany, phone: +49 221478 97641, +49 221478 5949, e-mail: xiaolei.ding09@gmail.com; Guilan Yang MD, Department of Cosmo-dermatology, Shenzhen Hospital, Southern Medical University, Guangdong 518100, China, phone: +86 15302653393, fax: +86-755-233-60302, e-mail: drgly2006@126.com

Received: 18.05.2018, accepted: 2.08.2018. 
the integration of ER function and the pathogenesis of various diseases associated with inflammation [11-13]. However, how ER changes and whether ERS implicates in PV pathogenesis have not been studied.

\section{Aim}

The main purpose of the present study was to determine ER and ERS in psoriatic keratinocytes. We examined the ultrastructure of ER using transmission electron microscopy (TEM) and discovered ER morphological changes in psoriatic keratinocytes, indicative of the increased ERS. Moreover, we investigated the expression and localization of ERS markers: binding immunoglobulin heavychain protein (BiP), C/EBP homologous protein ( $\mathrm{CHOP}$ ) and $\mathrm{X}$-box binding protein 1 (XBP-1) and found that the level of their expression is increased in psoriatic epidermis [14]. We further showed that the upregulated tumour necrosis factor $\alpha$ (TNF- $\alpha$ ) expression in PV epidermis indicates that both ERS and inflammation are activated in psoriatic keratinocytes.

\section{Material and methods}

\section{Human skin biopsies}

This was an open, prospective and parallel-arm clinical study. The whole procedure of the study has been approved by the Ethics Committee of Shenzhen Hospital, Southern Medical University (Guangzhou, China). Fourteen patients ( 9 males and 5 females) with an age range from 20 to 57 years were enrolled into this study. All the patients met the diagnostic criteria for PV in the disease activity period. Their psoriatic area and severity index (PASI) was from 9 to 32.7. The diagnosis of PV was based on clinical and histological features. These patients had not received any systemic or topical treatment apart from bland emollients at least 2 weeks before the study and no other systemic or skin diseases were accompanied. A detailed history and a complete physical examination were documented for all patients. Lesional and perilesional biopsies from the trunk or limbs were obtained by surgical resection. Control skin biopsies were collected from 14 age- and sex-matched healthy people treated for benign pigmented nevus of the trunk or limbs. The normal skin from the removed nevus tissues ( $3 \mathrm{~mm}$ away from the nevus site) was harvested and used as control. All consecutive biopsies were taken in an individual patient or control subject from the same anatomical area. Institutional review board approval and written informed consent for all subjects were obtained before entering into this study according to the Declaration of Helsinki.

\section{Electron microscopy}

The normal skin and psoriatic skin were cut into fragments, immediately fixed with $2.5 \%$ glutaraldehyde
(Roche) and followed by fixation with $1 \%$ osmium tetroxide. The fragments were contrasted with $0.4 \%$ uranyl acetate overnight, dehydrated with a graded alcohol series, and then embedded in Spurr resin. The blocks were sectioned with 1-3 cm thickness and stained with toluidine blue solution in $50 \%$ ethanol to determine the area of interest. Finally, ultrathin (90 nm thick) slices obtained with a diamond knife were collected using a 200-mesh grid and contrasted with $4 \%$ uranyl acetate solution and $0.4 \%$ lead citrate. All slices were examined under a transmission electron microscopy (JEM-1230, Leica, Germany).

\section{Immunohistochemical analysis}

Skin biopsy samples were fixed with $4 \%$ formalin, embedded in paraffin and sectioned for immunohistochemistry. Briefly, immunohistochemical staining was performed using antibodies against BiP (BS1154, Bioworld, USA), CHOP (BS1136, Bioworld, USA), XBP1 (BS6857, Bioworld, USA) and TNF- $\alpha$ (BS6000, Bioworld, USA) followed by incubation with horseradish peroxidase-conjugated secondary antibodies. DAB (brown) peroxidase substrates were used for visualization.

\section{Western blot analysis}

To separate epidermis and dermis, skin biopsies were immediately cut into strips with ophthalmic scissors and incubated in $1.2 \mathrm{U} / \mathrm{ml}$ dispase $/$ (Roche, Switzerland) for $30 \mathrm{~min}$ at $37^{\circ} \mathrm{C}$. The epidermal sheets were detached, washed and grinded with liquid nitrogen repeatedly. The epidermal tissues were lysed with radioimmunoprecipitation assay (RIPA) buffer (Beyotime, Nanjing, China). After centrifugation $\left(12,000 \mathrm{~g}, 15 \mathrm{~min}\right.$ at $\left.4^{\circ} \mathrm{C}\right)$, protein concentration was determined using BCA assay kit (Pierce, Rockford, IL, USA) and subjected to SDS-PAGE and blotted onto PVDF membrane (GE Healthcare, Beijing, China). After blocking (5\% non-fat milk), membrane was incubated with primary antibodies for $2 \mathrm{~h}$, washed and further incubated with horseradish peroxidase-conjugated secondary antibodies for 1.5 h. Protein bands were visualized using enhanced chemiluminescence (Amersham-Buchler, Braunschweig, Germany) and exposed to X-ray medical film (Kodak, Tokyo, Japan). GAPDH was used as the loading control.

\section{Statistical analysis}

Statistics was performed using PRISM software (GraphPad Prism 5). All results are expressed as mean \pm SD. Statistical differences between groups were determined according to one-way analysis of variance (ANOVA). $P$-values $<0.05$ were considered statistically significant.

\section{Results}

\section{Ultrastructural changes of the ER in PV epidermis}

Electron micrographs revealed that the epidermis of control subjects had similar basic features. The basal 
and spinous layers of keratinocytes were joined by desmosomes or interdigitations, containing a large nucleus with loose chromatin, the presence of mitochondria, and scarce endoplasmic reticulum cisternae and numerous free ribosomes (Figures 1 A1, 1 A2). Keratinocytes from adjacent tissue of PV patients were metabolically active and ER appeared similar to that of control normal skin
(Figures 1 B1, 1 B2). By contrast, the stratum spinosum of PV epidermis showed markedly metabolically active keratinocytes. Their nuclei were surrounded by granular endoplasmic reticulum with changes of ER morphology and the ER size expansion, suggesting that ER metabolism may be altered in psoriatic keratinocytes (Figures $1 \mathrm{C} 1,1 \mathrm{C} 2)$.
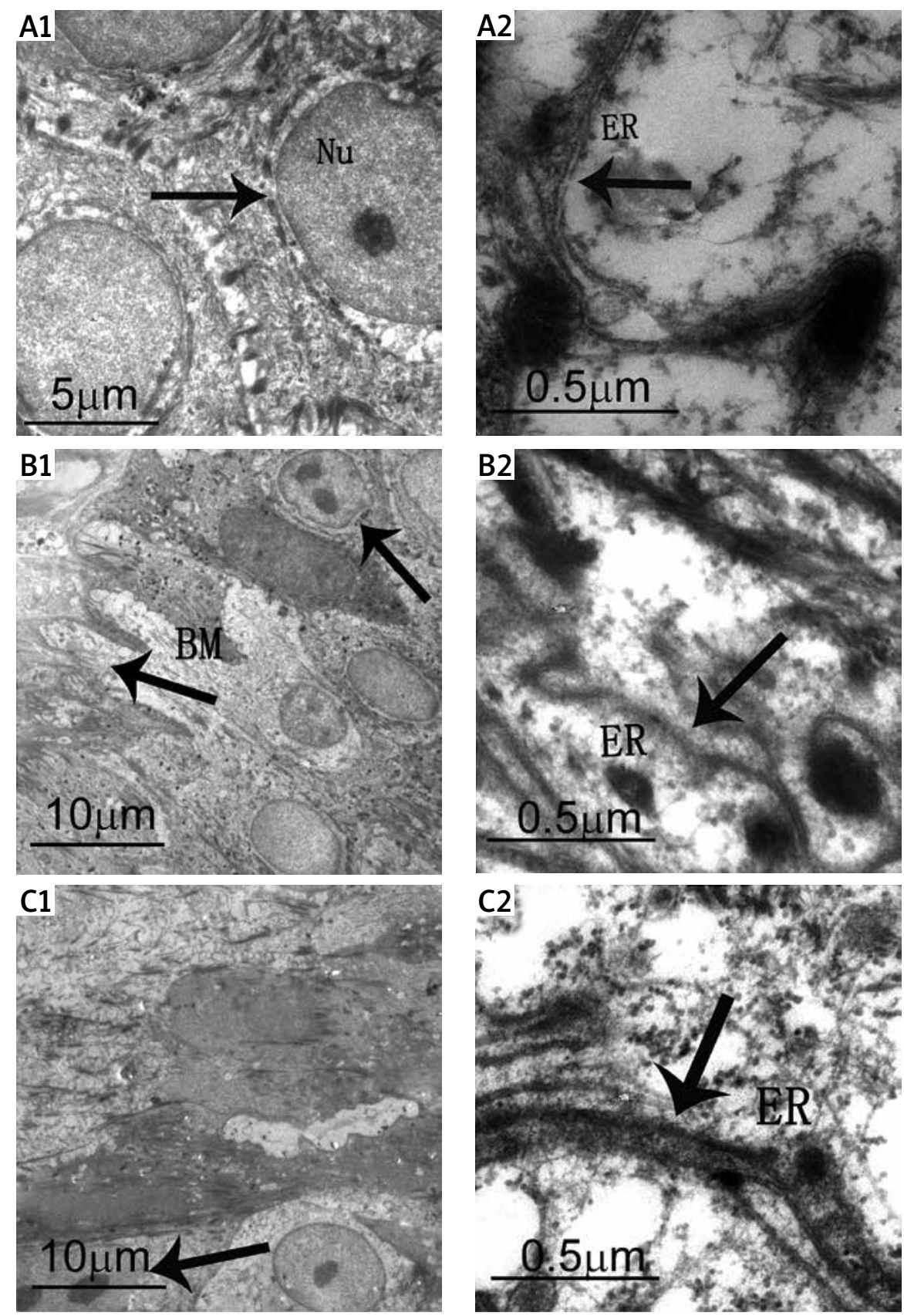

Figure 1. Ultrastructural analysis of ER in PV skin. A1 - Control skin. The arrow demonstrates basal cells connected with spinous layer cells by desmosomes or hemidesmosomes. Nu - nucleus. A2 - Control skin. The arrow demonstrates ER. A large number of free ribosomes are observable in keratinocytes. B1 - Adjacent skin tissue. The arrows demonstrate metabolically active cells and basement membrane (BM). B2 - Adjacent skin tissue. Normal ER are observable in the keratinocytes. C1 - Psoriasis skin tissue. The arrow demonstrates diving cells. C2 - Psoriasis skin tissue. The arrow demonstrates inflated ER 

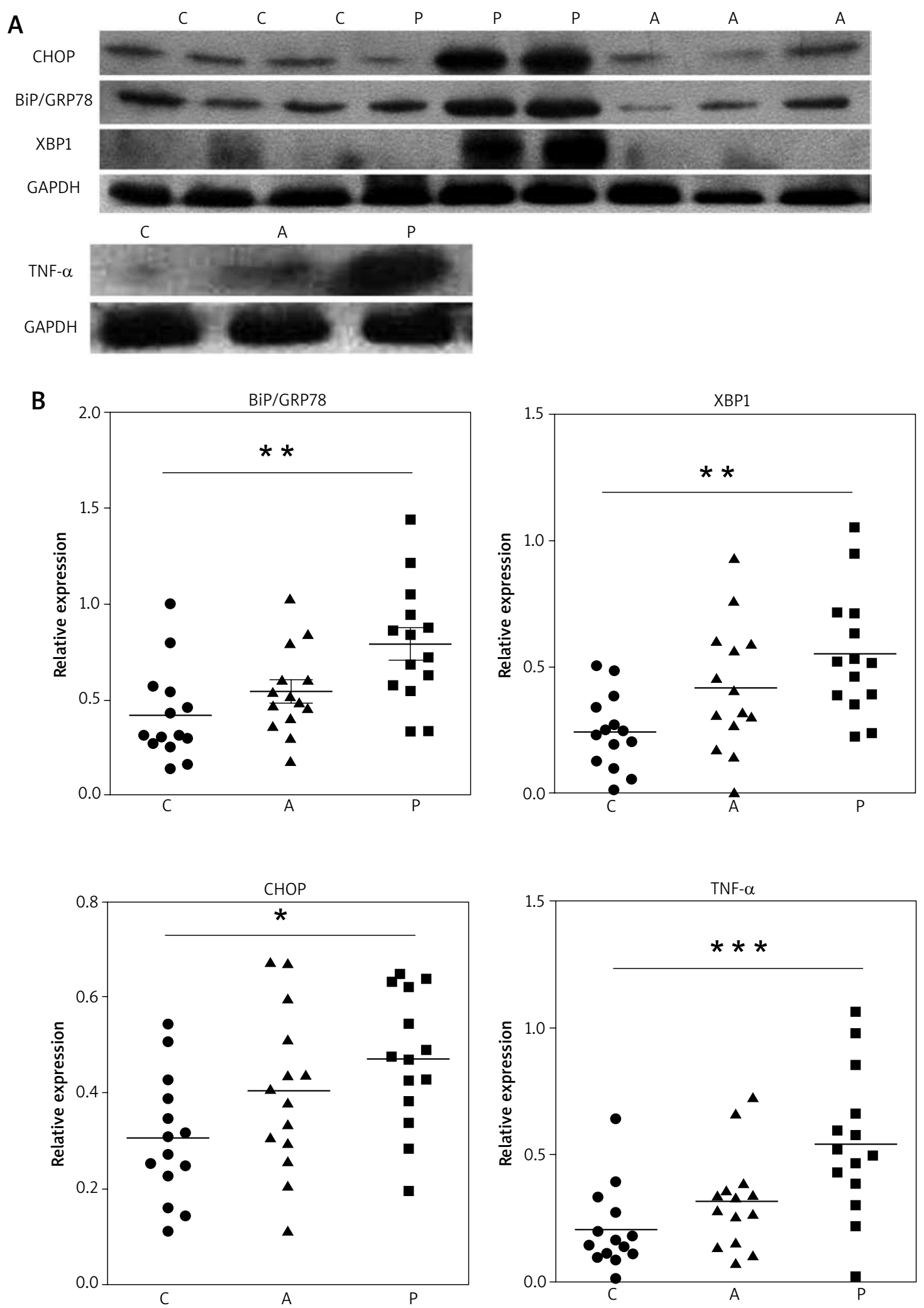

Figure 2. The expression of ERS-associated proteins is enhanced in psoriasis skin. A - Representative results of Western blot analysis. GADPH was used as a loading control. B - Quantitative results of protein expression basing on the intensity of Western blot assay as shown in panel A. Each dot represents one sample $C$ - control normal skin, $P$ - psoriasis $A$ - adjacent tissue. ${ }^{* * *} P<0.001$, ${ }^{* *} p<0.01,{ }^{*} p<0.05$. 


\section{Increased expression of BiP, CHOP, XBP1} and TNF- $\alpha$ proteins in PV epidermis

To identify whether PV keratinocytes had an increased ERS or activated UPR, we performed Western blot analysis with extracts of epidermis from normal skin, perilesional adjacent tissue, and lesional PV tissue using antibodies against $\mathrm{BiP}, \mathrm{CHOP}$ and XBP-1(S) (Figure 2 A). The expression of BiP, XBP-1 and CHOP was significantly upregulated in PV epidermis compared with healthy controls, but only mildly increased in adjacent tissue (Figure 2 B). TNF- $\alpha$ expression was significantly upregulated in PV tissue and showed a similar pattern as UPR proteins (Figures 2 A, B). These results suggest that the UPR was activated in epidermal keratinocytes of PV lesional skin.

\section{Immunohistochemical analysis of BiP, CHOP, XBP1 and TNF- $\alpha$ expression in PV skin}

We further examined the expression and localization of BiP, XBP-1, CHOP and TNF- $\alpha$ in PV skin and the controls by immunohistochemical analysis. As shown in Figure 3, the expression of BiP in healthy controls was almost completely restricted to the epidermal basal layer cells. In contrast, diffuse expression and strong staining signal of BiP was observed within the hyperplastic epithelium psoriasis skin and its expression was also increased in the epidermis of perilesional adjacent skin. XBP1, a transcriptional factor which lacked the staining signal in the healthy control skin, was increased in expression by basal and spinous layer cells in psoriatic skin and mainly localized in the nuclei (Figure 3). Simi-
C

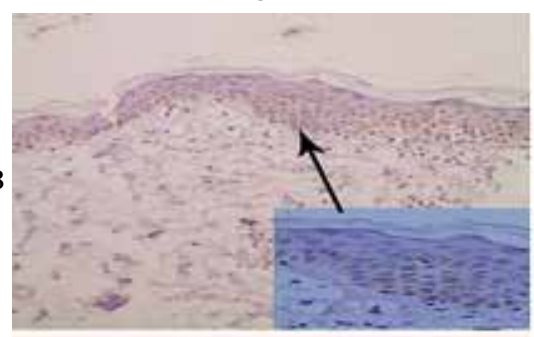

XBP1

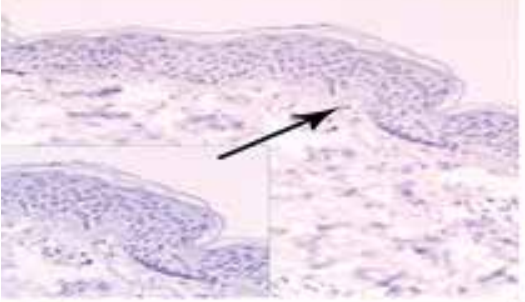

$P$
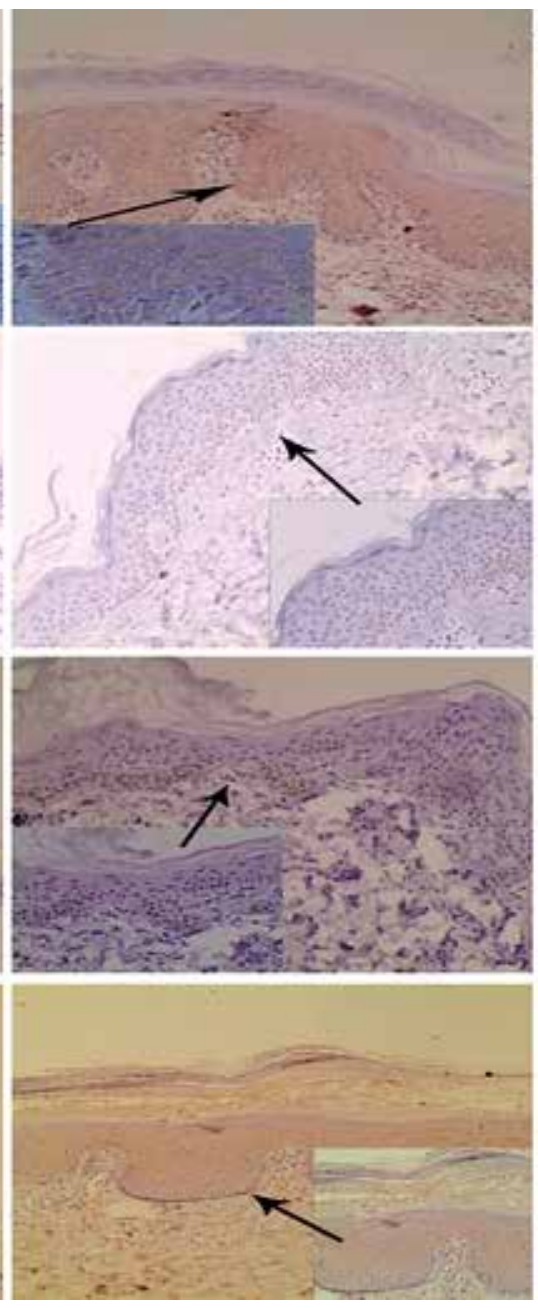

A
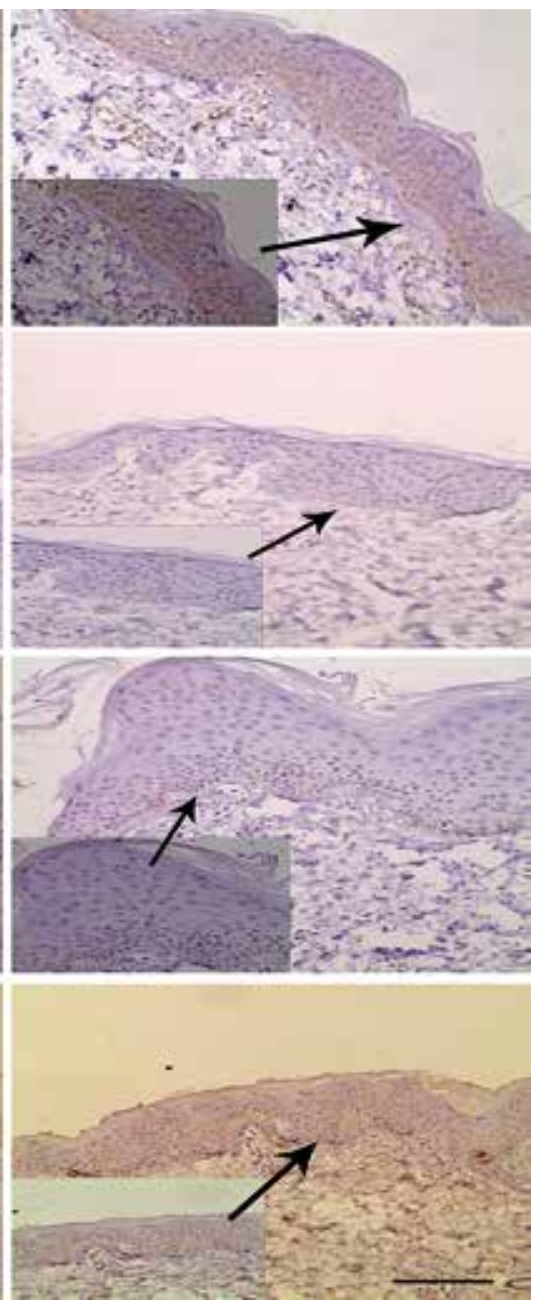

Figure 3. BiP, XBP-1, CHOP and TNF- $\alpha$ are overexpressed by the PV epidermis. Sections of control normal skin (C), psoriasis vulgaris tissue (P) and adjacent skin tissue (A) were stained with anti- BiP, XBP-1, CHOP and TNF- $\alpha$ antibodies respectively and counterstained with haematoxylin. Representative images are shown. Scale bar, $100 \mu \mathrm{m}$ 
larly, CHOP expression was markedly elevated in psoriatic skin and was limited in the basal spinous cell layer (Figure 3). TNF- $\alpha$ staining revealed that its expression was robustly increased in psoriatic skin, both the dermis and epidermis (Figure 3).

\section{Discussion}

Psoriasis is a complex inflammatory process mediated by innate and adaptive immunity and there is evidence that immune cells, cytokine and chemokine networks play important roles in psoriasis development [5, 15-17]. Although the contribution of immune response to psoriatic skin inflammation has been extensively characterized, it is still not clear how epidermal keratinocytes contribute to the disease pathology. Previous studies primarily described their dramatic changes in proliferation and aberrant differentiation of keratinocytes during the pathogenesis of psoriasis. Recent studies demonstrated that keratinocytes also exhibit a potent capacity to initiate skin inflammation to trigger the onset of inflammatory response in psoriasis [7]. Thus, by further identifying their abnormal behaviours and intrinsic mechanisms, those initiating skin inflammations are crucial for understanding the pathogenesis of psoriasis.

In the current study, we found that the ER in PV keratinocytes was ultrastructurally abnormal, implying the existence of ERS. We also demonstrated the increased expression of ERS-associated proteins: BiP, CHOP and XBP1 in the PV epidermis. The results suggested that ERS might be linked with the development and progression of psoriasis pathogenesis. To our knowledge, this is the first report on augmented ERS in PV keratinocytes. In line with our observations, a recent study has reported that ERS is activated in the dermal macrophages and endothelial cells under diabetic obese conditions [18]. Because of the high level of cytokine production by macrophages, ERS is supposed to play a systemic role in the psoriasiform inflammation of the diabetic skin [18]. In contrast, Sugiura [19] et al. showed that psoriasis vulgaris tissues had a low expression of BiP. The different observation with the report here might be rather methodical, as it only examined BiP expression by immunohistochemical staining with limited skin biopsies $(n=3)$ [19].

ERS has been implicated in the pathogenesis of many types of inflammatory and autoimmune diseases [20]. There are several potential avenues through which ER function is linked to inflammatory signalling [13, 21, 22]. For example, Deng et al. reported that both the IRE1a and PERK branches of the UPR can lead to activation of the

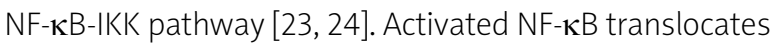
into the nucleus and switches on the expression of various genes involved in inflammatory pathways, such as IL-1 and TNF- $\alpha$. ERS also activates the cleavage of ERresident transcription factor, $\mathrm{CREBH}$, to induce a systemic inflammatory response [22]. Recently, the ATF6 branch of the UPR has also been linked to NF-KB-IKK signalling [25] suggesting that specific inflammatory triggers may signal through different branches of the UPR. Moreover, the UPR has been reported to cause increased expression of pro-inflammatory factors such as IL-8, IL-6, MCP-1, and TNF- $\alpha$ [20]. TNF- $\alpha$ is an important pro-inflammatory cytokine and plays an essential role in the pathogenesis of PV. In our study, we observed the correlated expression pattern between ERS- proteins and TNF- $\alpha$. Therefore, we presumed that the increased TNF- $\alpha$ level may at least partially result from ERS of PV and the close link between ERS and inflammation is a likely contributor to the pathogenesis of PV, given the critical role of inflammation in psoriasis.

\section{Conclusions}

Taken together, our study provides the first observations of increased ERS in PV skin. The findings suggested that ERS may be an important mediator of inflammation through the induction of TNF- $\alpha$ expression and may play a crucial role in the pathogenesis of PV. Future studies will focus on whether altering ERS signalling could be used as a treatment strategy for psoriasis.

\section{Acknowledgments}

The authors thank Professor Guang-Ming Zhou for assistance. This study was supported by the Institute of Modern Physics of the Chinese Academy of Sciences.

\section{Conflict of interest}

The authors declare no conflict of interest.

\section{References}

1. Parisi R, Symmons DPM, Griffiths CEM, Ashcroft DM. Global epidemiology of psoriasis: a systematic review of incidence and prevalence. J Investig Dermatol 2013; 133: 377-85.

2. Griffiths CE, Barker JN. Pathogenesis and clinical features of psoriasis. Lancet 2007; 370: 263-71.

3. Ayala-Fontanez N, Soler DC, McCormick TS. Current knowledge on psoriasis and autoimmune diseases. Psoriasis (Auckl) 2016; 6: 7-32.

4. Hawkes JE, Chan TC, Krueger JG. Psoriasis pathogenesis and the development of novel targeted immune therapies. J Allergy Clin Immunol 2017; 140: 645-53.

5. Nickoloff BJ, Xin H, Nestle FO, Qin JZ. The cytokine and chemokine network in psoriasis. Clin Dermatol 2007; 25: 56873.

6. Lowes MA, Suarez-Farinas M, Krueger JG. Immunology of psoriasis. Ann Rev Immunol 2014; 32: 227-55.

7. Kumari S, Bonnet MC, Ulvmar MH, et al. Tumor necrosis factor receptor signaling in keratinocytes triggers interleukin24-dependent psoriasis-like skin inflammation in mice. Immunity 2013; 39: 899-911.

8. Ron D, Walter P. Signal integration in the endoplasmic reticulum unfolded protein response. Nature Rev Mol Cell Biol 2007; 8: 519-29. 
9. Todd DJ, Lee AH, Glimcher LH. The endoplasmic reticulum stress response in immunity and autoimmunity. Nat Rev Immunol 2008; 8: 663-74.

10. Hotamisligil GS. Endoplasmic reticulum stress and the inflammatory basis of metabolic disease. Cell 2010; 140: 900-17.

11. Tabas I. The role of endoplasmic reticulum stress in the progression of atherosclerosis. Circ Res 2010; 107: 839-50.

12. Baban B, Liu JY, Mozaffari MS. Endoplasmic reticulum stress response and inflammatory cytokines in type 2 diabetic nephropathy: role of indoleamine 2,3-dioxygenase and programmed death-1. Exp Mol Pathol 2013; 94: 343-51.

13. Zhang K, Kaufman RJ. From endoplasmic-reticulum stress to the inflammatory response. Nature 2008; 454: 455-62.

14. Samali A, Fitzgerald U, Deegan S, Gupta S. Methods for monitoring endoplasmic reticulum stress and the unfolded protein response. Int J Cell Biol 2010; 2010: 830307.

15. Botti E, Spallone G, Caruso R, et al. Psoriasis, from pathogenesis to therapeutic strategies: IL-21 as a novel potential therapeutic target. Curr Pharm Biotechnol 2012; 13: 1861-7.

16. Mease PJ. Inhibition of interleukin-17, interleukin-23 and the TH17 cell pathway in the treatment of psoriatic arthritis and psoriasis. Curr Opin Rheumatol 2015; 27: 127-33.

17. Nickoloff BJ, Wrone-Smith T. Injection of pre-psoriatic skin with CD4+ T cells induces psoriasis. Am J Pathol 1999; 155: 145-58.

18. Shimoura N, Nagai H, Fujiwara S, et al. Exacerbation and prolongation of psoriasiform inflammation in diabetic obese mice: a synergistic role of CXCL5 and endoplasmic reticulum stress. J Investig Dermatol 2018; 138: 854-63.

19. Sugiura K, Muro Y, Futamura K, et al. The unfolded protein response is activated in differentiating epidermal keratinocytes. J Investig Dermatol 2009; 129: 2126-35.

20. Li Y, Schwabe RF, DeVries-Seimon T, et al. Free cholesterol-loaded macrophages are an abundant source of tumor necrosis factor-alpha and interleukin-6: model of NF-kappaB- and map kinase-dependent inflammation in advanced atherosclerosis. J Biol Chem 2005; 280: 21763-72.

21. Luo Y, Li SJ, Yang J, et al. HMGB1 induces an inflammatory response in endothelial cells via the RAGE-dependent endoplasmic reticulum stress pathway. Biochem Biophys Res Commun 2013; 438: 732-8.

22. Zhang K, Shen $\mathrm{X}$, Wu J, et al. Endoplasmic reticulum stress activates cleavage of CREBH to induce a systemic inflammatory response. Cell 2006; 124: 587-99.

23. Deng J, Lu PD, Zhang Y, et al. Translational repression mediates activation of nuclear factor kappa B by phosphorylated translation initiation factor 2. Mol Cell Biol 2004; 24: 10161-8.

24. Hu P, Han Z, Couvillon AD, et al. Autocrine tumor necrosis factor alpha links endoplasmic reticulum stress to the membrane death receptor pathway through IRElalpha-mediated NF-kappaB activation and down-regulation of TRAF2 expression. Mol Cell Biol 2006; 26: 3071-84.

25. Yamazaki H, Hiramatsu N, Hayakawa K, et al. Activation of the Akt-NF-kappaB pathway by subtilase cytotoxin through the ATF6 branch of the unfolded protein response. J Immunol 2009; 183: 1480-7. 